\title{
Virtual Compton Scattering at MAMI
}

\author{
N. d'Hose ${ }^{\mathrm{a}}$ \\ CEA-Saclay, DAPNIA/SPhN, F91191 Gif-sur-Yvette Cedex, France \\ / \\ Published online: 17 May 2006 - C Società Italiana di Fisica / Springer-Verlag 2006
}

\begin{abstract}
Virtual Compton Scattering (VCS) off the proton is a recent field of investigation of the nucleon structure. VCS at threshold gives access to the Generalized Polarizabilities (GPs) of the proton. The qualities of both the beam and the high-resolution spectrometers available at the Mainz Microtron MAMI allowed us to perform at first such delicate experiments. This paper deals with different experiments dedicated to the GPs measurements. They are realized without and with polarization, below and just above pion threshold.
\end{abstract}

PACS. 13.60.Fz Elastic and Compton scattering - 14.20.Dh Protons and neutrons - 25.30.Rw Electroproduction reactions

\section{Virtual Compton Scattering and polarizabilities}

One of the main challenges of hadronic physics in the regime of strong (non-perturbative) QCD is to identify the relevant degrees of freedom of the nucleon. Though the small distance structure is rather well described by point-like quarks and gluons, its structure at larger distance is not so well understood. There exist many models ranging from constituent quark models to chiral models.

Polarizabilities are one of the fundamental observables to describe the internal structure of the nucleon and they have been investigated with real Compton scattering (RCS) since the early 1950s. As the light scattering on atmospheric atoms which gives the well known Rayleigh effect for blue skies and red sunsets through oscillation of the electrons inside the atoms, real Compton scattering sheds light on the nucleon structure. This is clearly illustrated in a common definition of the electric polarizability $\alpha_{E}$ in a non relativistic approach at the first-order perturbation for an applied electric dipole moment $D$ :

$$
\alpha_{E}=2 \Sigma_{N^{\star} \neq N} \frac{\left|\left\langle N^{\star}\left|D_{z}\right| N\right\rangle\right|^{2}}{E_{N^{\star}}-E_{N}} .
$$

In this formula $N^{\star}$ indicates each nucleon resonance. The polarizability is then sensitive to all the excitation spectrum of the nucleon (even if the low energy of the perturbating photon does not allow the real formation of the nucleon resonances).

The Mainz laboratory has a long tradition in this field. Several experiments have been dedicated to the determination of proton, neutron or pion polarizabilities. Today

\footnotetext{
a e-mail: ndhose@cea.fr
}

the world global average of the electric $\left(\alpha_{E}\right)$ and magnetic $\left(\beta_{M}\right)$ polarizabilities on the proton is based on an experimental study investigated at MAMI with the tagged real photon beam [1].

$\alpha_{E}=(11.9 \pm 0.5$ (stat.) $\mp 1.3$ (syst. $\left.) \pm 0.3(\bmod ).\right) 10^{-4} \mathrm{fm}^{3}$, $\beta_{M}=(1.2 \pm 0.7$ (stat. $) \pm 0.3$ (syst.) $\left.\pm 0.4(\bmod ).\right) 10^{-4} \mathrm{fm}^{3}$.

We can note the small size of the polarizabilities which reveals the feature that the nucleon is strongly bound. For comparison the electric polarizability of the hydrogen atom is of the order of the atomic volume, and the electric polarizability of the proton $\alpha_{E}$ is only 0.05 per cent of its volume. Furthermore the magnetic polarizability is still smaller, one tenth of the electric polarizability.

Virtual Compton scattering (VCS) off the proton refers to the reaction $\gamma^{*} p \rightarrow p \gamma$, where $\gamma^{*}$ stands for an incoming virtual photon of four-momentum squared $Q^{2}$. This reaction is experimentally accessed through photon electroproduction $e p \rightarrow e p \gamma$. The corresponding Feynman diagram is indicated in fig. 1.

In the 1960 s the VCS appeared as a rather unwanted contribution to radiative corrections to electron scattering on a proton [2]. It was mentioned as proton Bremsstrahlung. In 1974 Arenhövel and Drechsel [3], from the Institut für Kernphysik at Mainz, considered the VCS for the first time as a good way to measure generalized polarizabilities (GPs). Only in 1995 with the new generation of facilities of high duty cycle to investigate exclusive reactions, and with new theoretical concepts it regained interest.

The general theoretical framework for VCS at threshold has been extensively described by Guichon et al. [4,5] and the Mainz theoretical group conducted by Drechsel [6, 7]. VCS reaction at threshold means that the produced 


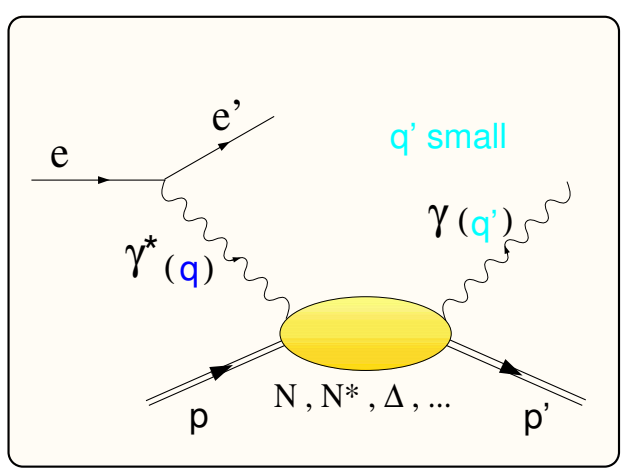

Fig. 1. The VCS graph for the proton.

photon has a small enough momentum or that its electric (E) and magnetic (M) fields look constant over the size of the nucleon. In the following the three-momenta absolute values of the virtual and real photons in the photon-proton center of mass (c.m.) system are noted $q$ and $q^{\prime}$, respectively, and vary independently, this is in contrast with real Compton scattering where $q=q^{\prime}$. Here the low momentum $\mathrm{q}^{\prime}$ of the produced real photon defines the size of the electromagnetic (EM) perturbation, while the momentum of the virtual photon q (or the four-momentum squared $\left.Q^{2}\right)$ sets the scale of the observation of the nucleon internal structure.

In the low momentum regime the reaction can be interpreted as electron scattering on a nucleon placed in a quasi-constant applied EM field [5]. The induced motion of the nucleon as a whole can be eliminated thanks to a low-energy theorem [8], so one is left with the deformation, due to the applied field, of the nucleon internal currents $\delta J^{\mu}(r)$ and the electron scattering measures its Fourier transform $\delta J^{\mu}(Q)$. To lowest order in $\alpha_{Q E D}, \delta J^{\mu}(Q)$ is linear in the applied field and the 6 coefficients of proportionality are the GPs $[4,5,6,7]$. When $Q^{2}=0$ two of them reduce to the usual polarizabilities $\alpha_{E}$ and $\beta_{M}$ measured in real Compton scattering. Analogously to the form factors for elastic scattering, which describes the charge and magnetization distributions, VCS gives access to the deformation of these distributions by an external EM field, and will yield valuable information about the non perturbative structure of the nucleon.

This can be illustrated by a very naive picture of the polarizabilities which are the results of an electromagnetic perturbation applied to the nucleon components. An electric field moves positive and negative charges inside the proton in opposite directions. The induced electric dipole moment is proportional to the electric field, and the proportionality coefficient is the electric polarizability $\alpha_{E}$ which measures the rigidity of the proton. A magnetic field acts differently on the quarks and the pion cloud. The quarks (of spin 1/2) align their magnetic moment parallel to the magnetic field giving the strong magnetic excitation of the $\Delta(1232)$ resonance. The pions are at low energy, an essential element of the structure of the nucleon notably at its surface giving the famous representation of a pion cloud surrounding the nucleon. The pions (of spin 0) distributed at the surface of the proton, will generate eddy currents.
When the magnetic field is applied, they are modified in such a way that the induced magnetic moment is antiparallel to the magnetic field (Lenz law). Quarks and pions thus give rise to two different contributions: - para and diamagnetic — or — resonant and non resonant contributions to the magnetic polarizability $\beta_{M}$.

At present the GPs have been calculated in the framework of various theoretical models $[9,10,11,12,13,14,15]$ yielding quite different results with regard to both their absolute value and their $Q^{2}$ dependence. Figure 2 presents different theoretical predictions as functions of $Q^{2}$ :

- Non relativistic constituent quark model (CQM) is based on the assumption that baryons are composed of three massive quarks moving within a harmonic oscillator confining potential and additional hyperfine interactions. One of its success is to explain most of the observed nucleon resonance mass spectrum. Calculations have been performed in this framework by Guichon, Liu and Thomas [4,9] and Pasquini, Scherer and Drechsel [10].

- Phenomenological approach can be realized with an effective Lagrangian model (ELM). Such a calculation has been performed by Vanderhaeghen [11] which includes the effects of all the first nucleon resonances and $\pi^{0}$ exchange in the $t$ channel.

These two first kinds of model describe well all the resonant contributions, but not the non-resonant one. Their limitation is that they have no relationship to chiral symmetry. This is an important property of QCD which governs much of low-energy hadron physics. The pion is the Goldstone boson of spontaneously broken chiral symmetry, and plays a very special and major role at low energy. The two next groups of calculations respect chiral symmetry.

- A simple model to describe interaction of Dirac particles with a chiral field is the linear sigma model (LSM) in the limit of an infinite sigma mass. Though this model is not a very realistic description of the nucleon, nevertheless it fulfills all the relevant symmetries like Lorentz, gauge and chiral invariance. A complete calculation of all the one-loop diagram contributions (for the photon interaction with a nucleon-pion system) has been performed by Metz and Drechsel [12].

- Chiral perturbation theory (ChPT) is a very systematic and consistent approach with a most general Lagrangian based on QCD symmetries. Heavy-baryon chiral perturbation theory allows for a systematic perturbative expansion in powers of small parameters (no- ted $p$ ) as quark masses, inverse of hadron masses or external momenta. Hemmert, Holstein, Knöcklein and Scherer [13] have performed a third order $\mathcal{O}\left(p^{3}\right)$ calculation for all the GPs while Kao and Vanderheaghen [16] have performed a fourth order $\mathcal{O}\left(p^{4}\right)$ calculation but only for the spin polarizabilities which exclude predictions for $\alpha_{E}$ and $\beta_{M}$. Nevertheless previous calculations for $\alpha_{E}\left(Q^{2}\right)$ and $\beta_{M}\left(Q^{2}\right)$ have been realized at $Q^{2}=0$ by Bernard, Kaiser, Schmidt and Meissner [17] including all terms to order $\mathcal{O}\left(p^{4}\right)$ 
Table 1. prediction at $Q^{2}=0$ in the heavy baryon chiral perturbation theory.

\begin{tabular}{cc}
\hline Calculation at $\mathcal{O}\left(p^{3}\right)[13]$ & Calculation at $\mathcal{O}\left(p^{4}\right)[17]$ \\
\hline$\alpha_{E}=12.5 \times 10^{-4} \mathrm{fm}^{3}$ & $\alpha_{E}=10.5 \times 10^{-4} \mathrm{fm}^{3}$ \\
$\beta_{M}=1.25 \times 10^{-4} \mathrm{fm}^{3}$ & $\beta_{M}=3.5 \times 10^{-4} \mathrm{fm}^{3}$ \\
\hline
\end{tabular}
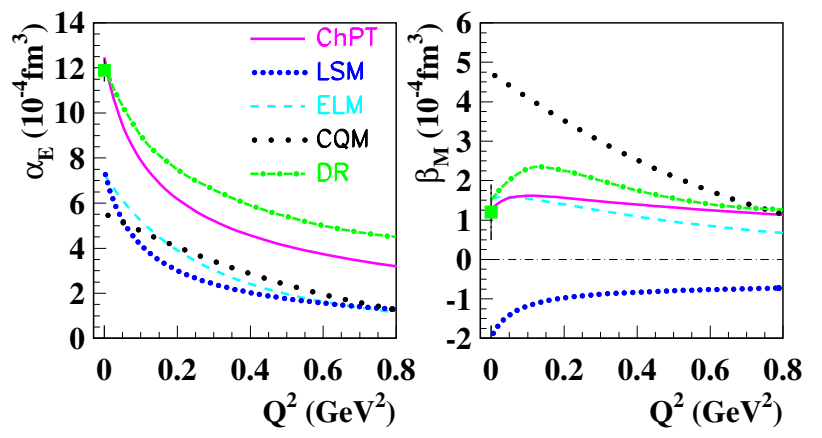

Fig. 2. Evolution of the electric and magnetic polarizabilities with $Q^{2}$. Experimental results [1] at $Q^{2}=0$ (with only statistical errors) and five theoretical predictions CQM [10], ELM [11], LSM [12], ChPT [13] and DR [15] are reported. See the text for comments.

and also $\Delta(1232)$-resonance contribution via counter terms. They found that in the case of the magnetic polarizability, a large positive contribution from the $\Delta(1232)$-resonance is largely canceled by a negative pionic contribution, which gives a rather small resulting value. The results at $Q^{2}=0$ are reported in table 1 .

Another consistent and unified approach for RCS and VCS has been given by the Mainz theoretical group through the dispersion relation formalism (DR) $[14,15]$ which connects the low energy nucleon structure quantities as polarizabilities to the nucleon excitation spectrum. A more detailed description will be given in the next section as this formalism is also used to extract the GPs.

In fig. 2 we observe a relatively sharp fall-off of the electric polarizability with increasing momentum transfer $Q^{2}$, while we can remark for the HBChPT, DR and ELM models a rise of the magnetic polarizability at very low transfer and then a decrease at larger transfer. This remarkable effect has its origin in the dominance of diamagnetism caused by the pion cloud at long distance and the dominance of paramagnetism due to a quark core at short distance. It is thus clear that the GPs are sensitive to the respective role of quark and pion degrees of freedom and as such they are very valuable new observables to compare theory with experiment.

\section{The unpolarized experiments at threshold}

A pioneer VCS experiment has been realized at MAMI at $Q^{2}=0.33 \mathrm{GeV}^{2}[18]$ during the years 1995-6-7, and then two experiments have been performed in two other

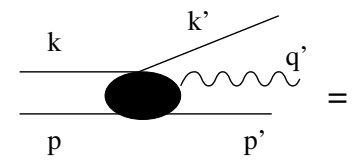

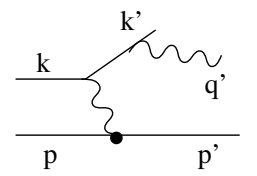

(a)

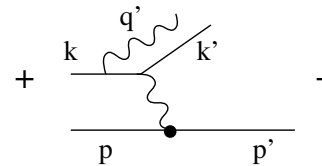

(b)

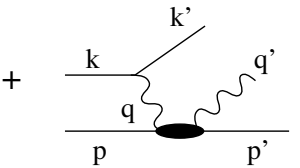

(c)
Fig. 3. The $p\left(e, e^{\prime} p\right) \gamma$ reaction. The initial, final electron and initial, final proton quadri-momenta are $k, k^{\prime}$ and $p, p^{\prime}$ respectively. The final photon quadri-momentum is $q^{\prime}$. In the one photon exchange approximation, a) and b) correspond to the Bethe-Heitler $(B H)$ process. c) corresponds to the Virtual Compton Scattering (VCS) process. We note $q$ the quadrimomentum of the virtual photon exchanged in the VCS process, that is $q=k-k^{\prime}\left(Q^{2}=-q^{2}\right)$.

complementary kinematical regimes at Jefferson Lab. $\left(Q^{2}=1\right.$ and $\left.2 \mathrm{GeV}^{2}\right)[19]$ in 1998 and MIT-Bates $\left(Q^{2}=\right.$ $\left.0.05 \mathrm{GeV}^{2}\right)[20]$ in 2000 . They are long and delicate experiments and they rely on a careful analysis of the data.

\subsection{Theoretical framework}

As it was mentioned the general theoretical framework for VCS is extensively presented in the following references [4, $5]$ and $[6,7]$. Only the relevant points for the analysis of these experiments will be discussed here. In the reaction $e p \rightarrow e p \gamma$, the final photon can be emitted either by the electrons, referring to the Bethe-Heitler $(\mathrm{BH})$ process, or by the proton, giving access to the VCS process (see fig. 3).

The $\mathrm{BH}$ process dominates and interferes strongly with the VCS process. The amplitude is the sum of the BH, Born and Non-Born amplitudes. The two last-named refer to the proton radiation: the Born amplitude depends only on the static properties of the proton (charge, mass) and elastic form factors, while the Non-Born amplitude contains dynamical internal structure information in terms of generalized polarizabilities. The differential cross section has the form

$$
\begin{aligned}
& \frac{\mathrm{d}^{5} \sigma^{e x p}}{\mathrm{dk}_{l a b}^{\prime}\left[\mathrm{d} \Omega_{e}\right]_{l a b}\left[\mathrm{~d} \Omega_{p}\right]_{C M}}= \\
& \frac{(2 \pi)^{-5}}{32 m}\left(\frac{\mathrm{k}_{l a b}^{\prime}}{\mathrm{k}_{l a b}}\right) \frac{\mathrm{q}^{\prime}}{\sqrt{s}} \times \mathcal{M} \equiv \phi \mathrm{q}^{\prime} \mathcal{M}
\end{aligned}
$$

wherein $\mathrm{k}_{l a b}, \mathrm{k}_{l a b}^{\prime}$ are the moduli of the incoming and outgoing electron momentum, respectively. The relevant kinematical variables of the problem are $\mathrm{q}$ and $\mathrm{q}^{\prime}$ previously defined; $\epsilon$ the virtual photon polarization; $\theta$ and $\varphi$ the two spherical angles indicating the CM real photon direction on a globe with the virtual photon as a pole. $\theta$ is the CM angle between the real and virtual photons while $\varphi$ represents the angle between the two electron plane and the photon-proton plane. In the precedent formula $\phi$ stands 
for a phase space factor. $\mathcal{M}$ is the coherent sum of the different amplitudes:

$$
\begin{aligned}
\mathcal{M} & =\frac{1}{4} \sum_{\text {spin }}\left|T^{B H}+T^{V C S}\right|^{2}, \\
& =\frac{1}{4} \sum_{\text {spin }}\left|T^{B H}+T^{\text {Born }}+T^{\text {NonBorn }}\right|^{2} .
\end{aligned}
$$

The low energy theorem (LET) from Low [8] states that in an expansion in powers of the real photon energy $q^{\prime}$ (but fixed arbitrary q), the first term of the amplitudes $T^{B H}$ and $T^{B o r n}$ is of the order $\mathrm{q}^{\prime-1}$ (well-known infrared divergence), while the first term of $T^{\text {NonBorn }}$ is of the order $\mathrm{q}^{1}$ :

$$
\begin{aligned}
T^{B H}+T^{\text {Born }} & =\frac{b_{-1}(\mathrm{q}, \epsilon, \theta, \varphi)}{\mathrm{q}^{\prime}}+\mathcal{O}\left(\mathrm{q}^{\prime 0}\right), \\
T^{N \text { onBorn }} & =b_{1}(\mathrm{q}, \epsilon, \theta, \varphi) \mathrm{q}^{\prime}+\mathcal{O}\left(\mathrm{q}^{\prime 2}\right) .
\end{aligned}
$$

Consequently in the low energy limit of the final photon, the cross section is independent of the dynamical nucleon structure [8], and can be evaluated using only the known $\mathrm{BH}$ and Born amplitudes. This can be summarized by the following equation:

$$
\begin{aligned}
\mathrm{d}^{5} \sigma^{\exp }\left(\mathrm{q}, \mathrm{q}^{\prime}, \epsilon, \theta, \varphi\right) & =\mathrm{d}^{5} \sigma^{B H+B o r n}\left(\mathrm{q}, \mathrm{q}^{\prime}, \epsilon, \theta, \varphi\right) \\
& +\phi \mathrm{q}^{\prime} \Psi_{0}(\mathrm{q}, \epsilon, \theta, \varphi)+\phi \mathcal{O}\left(\mathrm{q}^{\prime 2}\right)
\end{aligned}
$$

where $\mathrm{d}^{5} \sigma$ is a notation for the differential cross section $\mathrm{d}^{5} \sigma / \mathrm{dk}_{l a b}^{\prime}\left[\mathrm{d} \Omega_{e}\right]_{l a b}\left[\mathrm{~d} \Omega_{p}\right]_{C M} . \Psi_{0}(\mathrm{q}, \epsilon, \theta, \varphi)$ is the leading term in the expansion in powers of the real photon momentum $\mathrm{q}^{\prime}$. It corresponds to the interference between the term of order $\mathrm{q}^{\prime-1}$ in the $\mathrm{BH}+$ Born amplitude and the leading order term of order $\mathrm{q}^{1}$ in the Non-Born amplitude. It contains the dynamical internal structure information of the proton, parametrized by 6 generalized polarizabilities given for the electric and magnetic dipole radiation of the outgoing real photon.

We note $L\left(L^{\prime}\right)$ the initial (final) photon orbital angular momentum, $\rho\left(\rho^{\prime}\right)$ the type of multipole transition (0 for Coulomb, 1 for Magnetic, 2 for Electric), and $S$ the type of the transition at the nucleon side (non-spin-flip $S=0$ and spin-flip $S=1$ ). Assuming that the emitted real photon has low energy, we may use the dipole approximation $\left(L^{\prime}=1\right)$. For a dipole transition in the final state, parity and angular momentum conservations lead to $10 \mathrm{GPs}$ presented in table 2. Crossing symmetry and charge conjugation invariance provide 4 relations between the $10 \mathrm{GPs}$ and we are left with 6 independent GPs: 2 scalar $(S=0)$ and 4 spin-dependent $(S=1)$ polarizabilities, functions of q (or equivalently $\tilde{Q}^{2}=\left.Q^{2}\right|_{\mathrm{q}^{\prime}=0}$. See footnote ${ }^{1}$ ). The choice of 6 GPs is a arbitrary, and can be realized for example by the 6 surrounded GPs in table 2 .

In an unpolarized measurement, $\Psi_{0}(\mathrm{q}, \epsilon, \theta, \varphi)$ can be written as

$$
\begin{aligned}
\Psi_{0}(\mathrm{q}, \epsilon, \theta, \varphi) & =v_{1}(\theta, \varphi, \mathrm{q})\left(\mathbf{P}_{\mathbf{L L}}(\mathrm{q})-\mathbf{P}_{\mathbf{T T}}(\mathrm{q}) / \epsilon\right) \\
& +v_{2}(\theta, \varphi, \mathrm{q}) \mathbf{P}_{\mathbf{L T}}(\mathrm{q})
\end{aligned}
$$

${ }^{1} \tilde{Q}^{2}=\left.Q^{2}\right|_{\mathrm{q}^{\prime}=0}=2 m \cdot\left(\sqrt{m^{2}+\mathrm{q}^{2}}-m\right)$ where $m$ stands for the proton mass.
Table 2. List of the 10 GPs with the corresponding electromagnetic transitions. Their relation with the polarizabilities obtained in real Compton scattering are indicated. 6 GPs are independent. Our choice is a priori arbitrary, and is realized by the 6 surrounded GPs.

\begin{tabular}{cccc}
\hline EM transition & VCS GPS & & RCS polarizabilities \\
\hline & & $Q^{2} \rightarrow 0$ & \\
$\mathcal{M}^{(C 0 \rightarrow M 1) S=1}$ & $P^{(11,00) 1}$ & $\rightarrow$ & 0 \\
$\mathcal{M}^{(C 2 \rightarrow M 1) S=1}$ & $P^{(11,02) 1}$ & $\rightarrow$ & $-\sqrt{\frac{8}{27}} \frac{4 \pi}{e^{2}}\left(\gamma_{2}+\gamma_{4}\right)$ \\
$\mathcal{M}^{(M 1 \rightarrow M 1) S=0}$ & $P^{(11,11) 0}$ & $\rightarrow$ & $-\sqrt{\frac{8}{3}} \frac{4 \pi}{e^{2}}\left(\beta_{M}\right)$ \\
$\mathcal{M}^{(M 1 \rightarrow M 1) S=1}$ & $P^{(11,11) 1}$ & $\rightarrow$ & 0 \\
$\mathcal{M}^{(C 2, E 2 \rightarrow M 1) S=1}$ & $\hat{P}^{(11,2) 1}$ & $\rightarrow$ & $\neq 0$ \\
& & & \\
$\mathcal{M}^{(C 1 \rightarrow E 1) S=1}$ & $P^{(01,01) 1}$ & $\rightarrow$ & 0 \\
$\mathcal{M}^{(C 1 \rightarrow E 1) S=0}$ & $P^{(01,01) 0}$ & $\rightarrow$ & $-\sqrt{\frac{2}{3}} \frac{4 \pi}{e^{2}}\left(\alpha_{E}\right)$ \\
$\mathcal{M}^{(M 2 \rightarrow E 1) S=1}$ & $P^{(01,12) 1}$ & $\rightarrow$ & $-\frac{\sqrt{2}}{3} \frac{4 \pi}{e^{2}}\left(\gamma_{3}\right)$ \\
$\mathcal{M}^{(C 1, E 1 \rightarrow E 1) S=1}$ & $\hat{P}^{(01,1) 1}$ & $\rightarrow$ & $\neq 0$ \\
$\mathcal{M}^{(C 1, E 1 \rightarrow E 1) S=0}$ & $\hat{P}^{(01,1) 0}$ & $\rightarrow$ & $\neq 0$ \\
\hline
\end{tabular}

where $v_{1}(\theta, \varphi, \mathrm{q}), v_{2}(\theta, \varphi, \mathrm{q})$ are known kinematical factors. $\mathbf{P}_{\mathbf{L L}}(\mathrm{q}), \mathbf{P}_{\mathbf{T T}}$ (q), $\mathbf{P}_{\mathbf{L T}}$ (q) are structure functions related to the $\operatorname{GPs}(\mathrm{q})$ with some kinematical factors:

$$
\begin{aligned}
\mathbf{P}_{\mathbf{L L}} & =-2 \sqrt{6} m G_{E} P^{(01,01) 0} \\
\mathbf{P}_{\mathbf{T T}} & =3 G_{M} \mathrm{q}^{2}\left(\sqrt{2} P^{(01,12) 1}-P^{(11,11) 1} / \tilde{\mathrm{q}}_{0}\right) \\
\mathbf{P}_{\mathbf{L T}} & =\sqrt{\frac{3}{2}} \frac{m \mathrm{q}}{\tilde{Q}} G_{E} P^{(11,11) 0} \\
& +\frac{\sqrt{3}}{2} \frac{\tilde{Q}}{\mathrm{q}} G_{M}\left(P^{(11,00) 1}+\frac{\mathrm{q}^{2}}{\sqrt{2}} P^{(11,02) 1}\right)
\end{aligned}
$$

where $m$ stands for the proton mass, $G_{E}$ and $G_{M}$ denote the form factors evaluated at $\tilde{Q}^{2}$ and $\tilde{\mathrm{q}}_{0}$ is the $\mathrm{CM}$ virtual photon energy at $\mathrm{q}^{\prime}=0$. (See footnote ${ }^{2}$.)

The two structure functions $\mathbf{P}_{\mathbf{L L}}(\mathrm{q})-\mathbf{P}_{\mathbf{T T}}(\mathrm{q}) / \epsilon$ and $\mathbf{P}_{\mathbf{L T}}$ measured in an unpolarized VCS experiment are the sum of two contributions: one coming from the scalar or spin-independent polarizabilities and another one coming from the spin-dependent polarizabilities (which vanishes at $\left.Q^{2}=0\right)$.

\subsection{The MAMI experiment}

With the high luminosity and high duty cycle provided by the $855 \mathrm{MeV}$ Mainz Microtron MAMI it was possible to investigate the measurement of the small photon electroproduction cross section at threshold. Absolute cross sections $\mathrm{d}^{5} \sigma^{\exp }$ [18] have been measured at $Q^{2}=0.33 \mathrm{GeV}^{2}$ using the three-spectrometer facility [21] of the A1 collaboration at MAMI (see fig. 4). The scattered electron and the recoiling proton were detected in coincidence with two of the high-resolution magnetic spectrometers. The photon production process was selected by a cut on the missing mass

$$
{ }^{2} \tilde{\mathrm{q}}_{0}=m-\sqrt{m^{2}+\mathrm{q}^{2}} .
$$




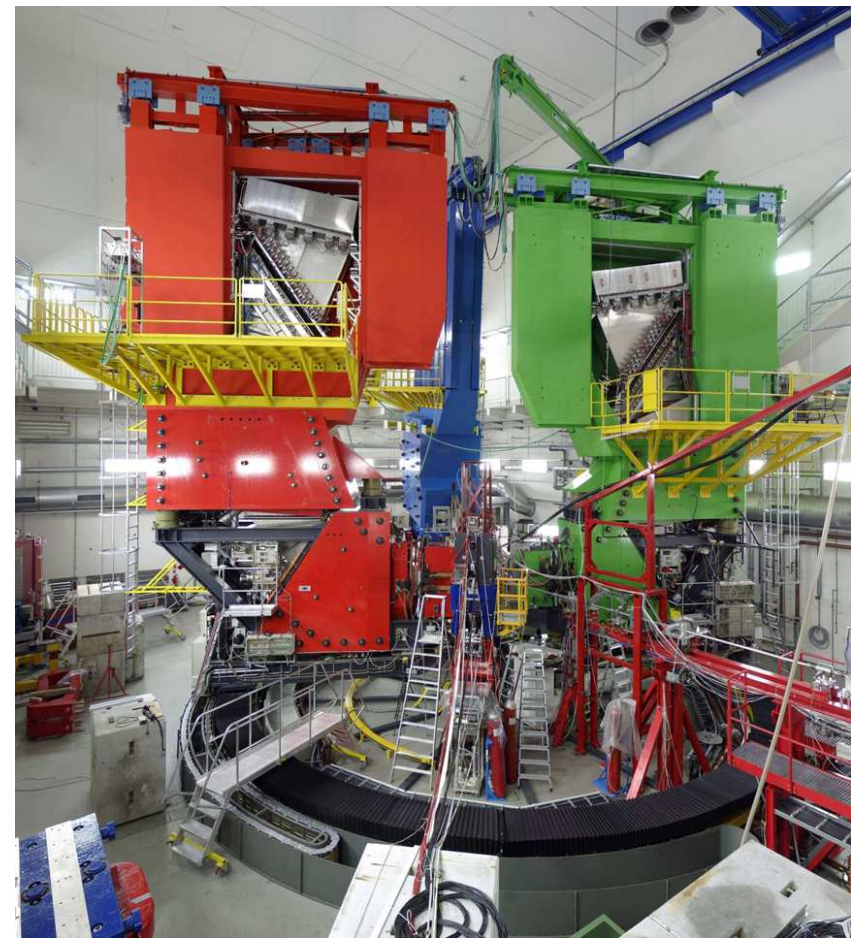

Fig. 4. The 3-high-resolution spectrometer facility of the A1 collaboration at MAMI.

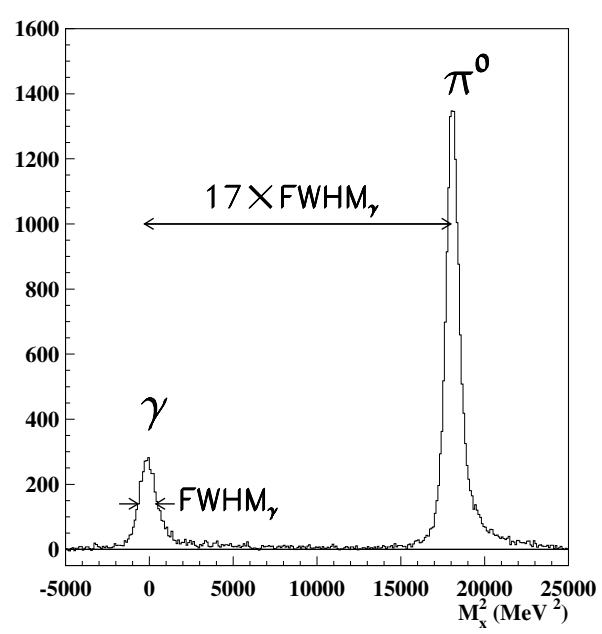

Fig. 5. Missing mass spectrum obtained for the setup at $\mathrm{q}^{\prime}=$ $111.5 \mathrm{MeV} / c$.

around zero, which was possible thanks to the excellent resolution of the facility (momentum resolution of $10^{-4}$ and angular resolution better than $3 \mathrm{mrad}$ ) (see fig. 5).

The aim of this first VCS experiment below pion threshold was to measure the five-fold differential cross sections in a wide photon angular range, at 5 values of the photon momentum $\mathrm{q}^{\prime}: 33.6,45,67.5,90$, and $111.5 \mathrm{MeV} / c$ (presentation in fig. 6). The 3 other kinematical variables were held fixed, namely the virtual photon momentum, $\mathrm{q}=600 \mathrm{MeV} / \mathrm{c}\left(\tilde{Q}^{2}=0.33 \mathrm{GeV}^{2}\right)$, the virtual photon polarization $\epsilon=0.62$. The out-of-plane angle $\varphi$ range is

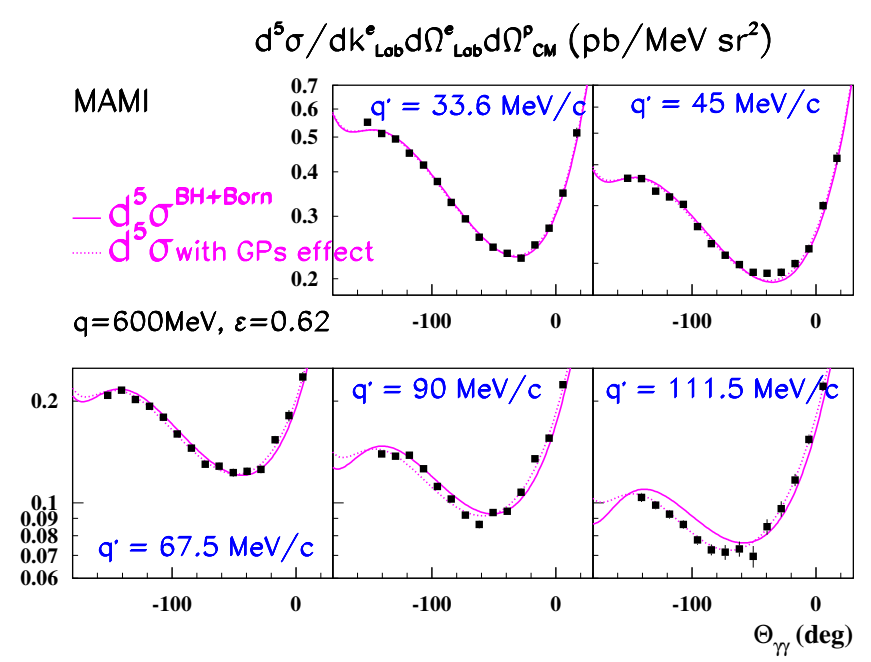

Fig. 6. Differential cross sections for the reaction $e p \rightarrow e p \gamma$ as a function of $\theta$ for fixed $\mathrm{q}, \epsilon, \varphi$ and for five values of the real photon momentum $\mathrm{q}^{\prime}$. The known part of the cross section $\mathrm{d}^{5} \sigma^{B H+B o r n}$, is presented by the solid lines. The experimental data points $\mathrm{d}^{5} \sigma^{\text {exp }}$ deviate from the solid lines as $\mathrm{q}^{\prime}$ increases - the effect of the proton polarizabilities. The dotted lines represent the expected cross sections with the effect of the polarizabilities measured by the two structure functions deduced from this experiment.

determined by the acceptance of the two spectrometers around $0^{\circ}$ and $180^{\circ}$. The spherical angles $\theta$ and $\varphi$ are defined such that $\varphi=0^{\circ}$ corresponds to the half plane containing the electron momenta. To ease the presentation the data are plotted with $\theta$ ranging from $-180^{\circ}$ to $+180^{\circ}$; the negative values corresponding in fact to $\varphi=180^{\circ}$. The wide range of $\theta$ from $-141^{\circ}$ to $+6^{\circ}$ covers the backward direction relative to the incoming and outgoing electrons. Here, the VCS contributions are dominant because the electron radiations $(\mathrm{BH})$ are emitted predominantly in the electron directions.

The cross sections $\mathrm{d}^{5} \sigma^{B H+B o r n}$ are presented by the solid lines in fig. 6. At small photon momentum $\mathrm{q}^{\prime}=33.6 \mathrm{MeV} / c$ the agreement between the radiatively corrected data and $\mathrm{d}^{5} \sigma^{B H+B o r n}$ is excellent, and the deviation from this known cross section increases when $\mathrm{q}^{\prime}$ increases, as expected from the effect of the proton polarizabilities. In order to determine accurately the polarizabilities, a careful analysis of possible systematic errors on the deviation is of particular importance. First the $\mathrm{BH}$ and Born contributions rely on the knowledge of the proton form factors. Consequently we also measured the absolute elastic scattering cross section for each kinematic setting of the VCS experiment. These measurements validate the use of the form factor parametrization from Höhler [22] at a precision better than $\pm 1 \%$. Second the radiative corrections, which are of the order of $20 \%$ of the cross section, have been evaluated by Vanderhaeghen et al. [23]. The systematic uncertainties are estimated to equal $\pm 2 \%$ for the calculation performed to order $\alpha^{4}$ in the VCS cross section. Third the luminosity and the detector efficiencies are controlled within an accuracy 


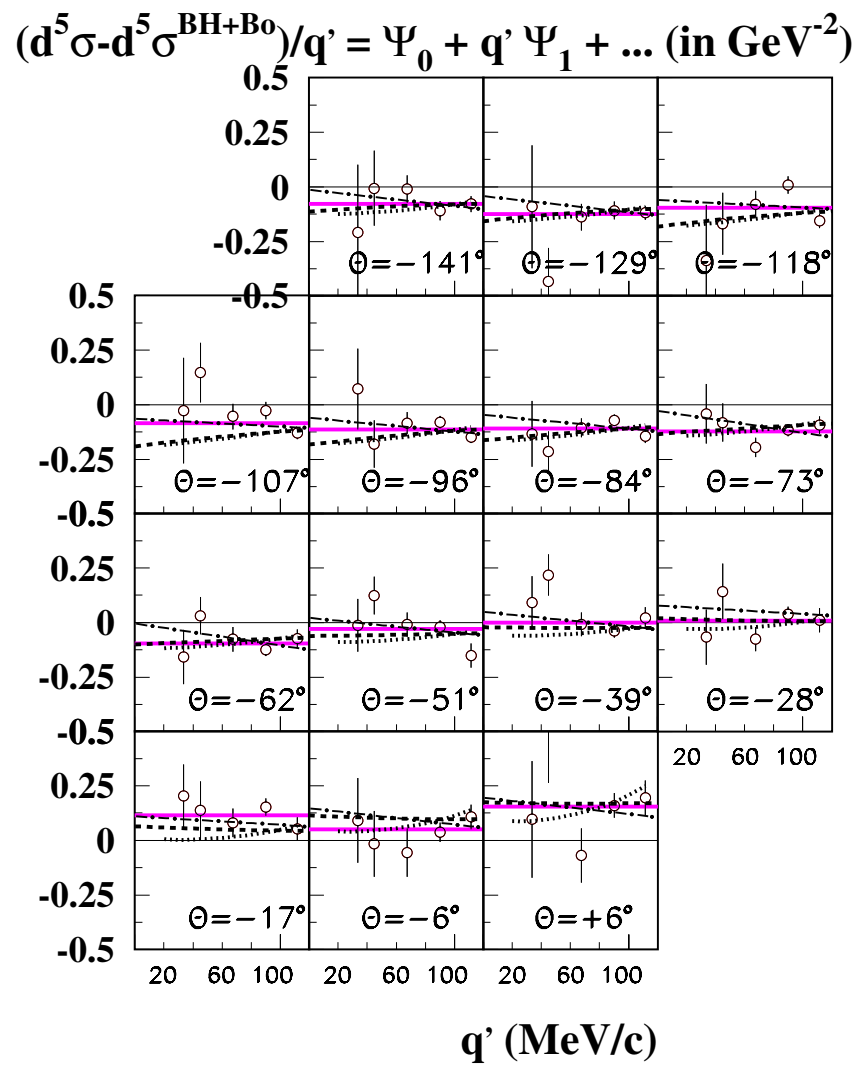

Fig. 7. $\left(\mathrm{d}^{5} \sigma-\mathrm{d}^{5} \sigma^{B H+B o r n}\right) / \phi \mathrm{q}^{\prime}$ studied as a function of the real photon momentum $\mathrm{q}^{\prime}$ for the 14 measured scattering angles $\theta$. The intercept at origin is $\Psi_{0}$. In a first method it is determined at each scattering angle $\theta$ by the mean value in the investigated real photon momentum range (solid line). The dash-dotted, dashed and dotted lines show evolutions in the framework of the 2nd, 3rd and 4th methods, respectively.

of $\pm 1 \%$, the solid angles are determined within an accuracy of $\pm 2 \%$ using a Monte Carlo [24] simulation which reproduces perfectly the missing mass spectra. All these uncertainties are constant over the angular range of the real photon and are controlled by the fairly good agreement between the radiatively corrected data and the predicted $\mathrm{BH}$ and Born cross section at small $\mathrm{q}^{\prime}$. However small imperfections in the spectrometer optic calibration which could provide distortion of the angular distributions are estimated to give a variation of cross section of $\pm 2.5 \%$.

Figure 7 shows the behavior of $\left(\mathrm{d}^{5} \sigma-\mathrm{d}^{5} \sigma^{B H+B o r n}\right) /$ $\phi \mathrm{q}^{\prime}$ as a function of the real photon momentum $\mathrm{q}^{\prime}$ for the 14 measured scattering angles $\theta$. The goal is to determine the intercept at origin (noted $\Psi_{0}$ in eq. (5)), and this figure illustrates the basic difficulty of this experiment that is the increase of the statistical errors when $q^{\prime}$ decreases. Four methods are then considered in the following.

First method based on the LET: As is apparent in fig. 7, there is no strong evolution with the real photon momentum. Therefore we make the hypothesis that there is no $\mathrm{q}^{\prime}$ dependence in $\left(\mathrm{d}^{5} \sigma-\mathrm{d}^{5} \sigma^{B H+B o r n}\right) / \phi \mathrm{q}^{\prime} . \Psi_{0}$ is then determined at each scattering angle $\theta$ by the mean value of the data at the 5 photon energies.

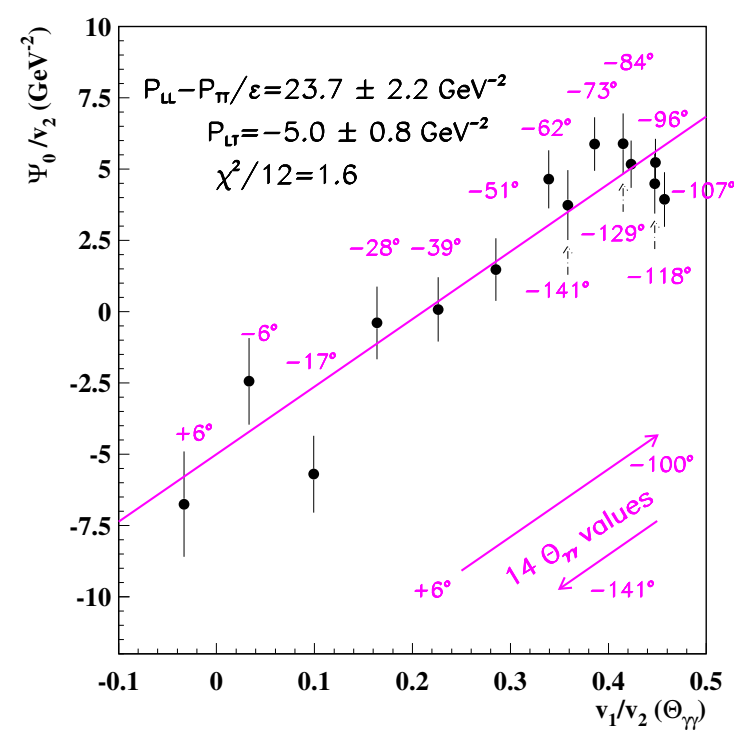

Fig. 8. Compilation of the complete data set (for the 14 angles) of $\Psi_{0} / v_{2}$ as a function of $v_{1} / v_{2}$. The data are reasonably well aligned; the errors indicated are statistical only. This allows to extract the two structure functions $\mathbf{P}_{\mathbf{L L}}-\mathbf{P}_{\mathbf{T T}} / \epsilon$ and $\mathbf{P}_{\mathbf{L T}}$ with statistical errors and the $\chi^{2}$ given.

Figure 8 presents the complete data set (for the 14 angles) of $\Psi_{0} / v_{2}$ as a function of $v_{1} / v_{2}$ (cf. eq. (6)). The data are reasonably well aligned, which suggests that the higher-order terms in the expansion of the cross section (cf. eq. (5)) are not so important. This good alignment for a wide angular range is also indicative of the consistency of the experimental data. We extract the two structure functions $\mathbf{P}_{\mathbf{L L}}-\mathbf{P}_{\mathbf{T T}} / \epsilon$ and $\mathbf{P}_{\mathbf{L T}}$ as the slope and intercept of a linear fit to the data (according to eq. (6)) [18].

Second method: We make the hypothesis of a linear evolution with the real photon momentum for each angle which is fitted to the data. The result is indicated by the dash-dotted line in fig. 7.

Third method: The $\mathrm{q}^{\prime}$ evolution is supposed to be governed by the interference between the complete $\mathrm{BH}$ and Born amplitudes considered at all order in the $\mathrm{q}^{\prime}$ expansion (complete eq. (3)) and the Non-Born amplitude truncated at the first order in the $\mathrm{q}^{\prime}$ expansion (truncated eq. (4)). The only parameters are a priori the 6 generalized polarizabilities contained in the first and only term of the considered Non-Born amplitude. They are adjusted with a best fit on the complete set of $14 \times 5$ data. In order to have a better convergence, the polarizability $P^{(01,01) 0}\left(Q^{2}=0.33 \mathrm{GeV}^{2}\right)$ is fixed by the result obtained in real Compton scattering scaled by the electric form factor and $P^{(11,02) 1}\left(Q^{2}=0.33 \mathrm{GeV}^{2}\right)$ is fixed at 0 (it corresponds to the quadrupolar deformation of the $\mathrm{N}-\Delta$ transition which is expected to be very small). The result of the fit for the 4 remaining polarizabilities is presented in table 3. This third method, mainly realized to justify a rather flat $\mathrm{q}^{\prime}$ evolution of $\left(\mathrm{d}^{5} \sigma-\mathrm{d}^{5} \sigma^{B H+B o r n}\right) / \phi \mathrm{q}^{\prime}$ presented by the dashed points in fig. 7 , allows one to 
Table 3. Results for the polarizabilities extracted in the third method. These results are compared to the heavy-baryon chiral perturbation theory (HBChPT) predictions [13].

\begin{tabular}{|l|l|c|c|c|}
\hline & & Third Method & HBChPT & Units \\
\hline $\mathbf{P}_{\mathbf{L L}}$ & $P^{(01,01) 0}$ & -0.0626 fixed & -0.056 & $\mathrm{fm}^{3}$ \\
\hline $\mathbf{P}_{\text {TT }}$ & $P^{(11,11) 1}$ & $+0.0048 \pm 0.0034$ & +0.001 & $\mathrm{fm}^{3}$ \\
& $P^{(01,12) 1}$ & $-0.0123 \pm 0.0026$ & -0.008 & $\mathrm{fm}^{4}$ \\
\hline $\mathbf{P}_{\mathbf{L T}}$ & $P^{(11,11) 0}$ & $-0.0384 \pm 0.0186$ & -0.034 & $\mathrm{fm}^{3}$ \\
& $P^{(11,00) 1}$ & $-0.157 \pm 0.070$ & -0.096 & $\mathrm{fm}^{2}$ \\
& $P^{(11,02) 0}$ & 0. fixed & +0.003 & $\mathrm{fm}^{4}$ \\
\hline
\end{tabular}

determine some spin polarizabilities with reasonable precision (notably $P^{(01,12) 1}$ ).

Fourth method using Dispersion Relations: This method was used after the publication [18] of the first VCS MAMI experiment at the sight of the other experiments of JLab and MAMI where a rather flat $\mathrm{q}^{\prime}$ evolution was not so obviously confirmed by the data. This method is based on the formalism of Dispersion Relations (DR) $[14,15]$ for the invariant VCS amplitudes and works below pion threshold as well as in the first resonance region. Assuming analyticity, crossing symmetry and an appropriate high-energy behavior, unsubtracted dispersion relations relate the real part of VCS amplitudes to an integral over the virtual photon energy of a function of their imaginary part. The imaginary part of a VCS amplitude is given by the sum of $\pi N$ intermediate states, computed from $\gamma^{*} N \rightarrow \pi N$ data (in the phenomenological MAID2000 analysis [25]), plus higher order contributions beyond $\pi N$. Moreover asymptotic contributions have also to be considered for two VCS amplitudes $\left(F_{1}\right.$ and $\left.F_{5}\right)$ which cannot fulfill unsubtracted dispersion relation framework. - The $t$-channel $\pi^{0}$ exchange and the knowledge of the $F_{\pi^{0} \gamma \gamma}$ form factor fix the asymptotic contribution to $F_{5}$ and determine completely the spin-dependent GPs.

- The asymptotic contribution of the amplitude $F_{1}$ related to the polarizability $P^{(11,11) 0}$ or $\beta_{M}\left(Q^{2}\right)$ originates from the $t$-channel $\pi \pi$ intermediate states. In a phenomenological analysis, this continuum is parametrized through the exchange of a scalar-isoscalar particle in the $t$-channel, i.e. an effective " $\sigma$ "-meson which gives rise to a diamagnetic contribution. The asymptotic part and the dispersive contributions beyond $\pi N$ are estimated using a dipole parametrization of the difference:

$$
\beta_{M}\left(Q^{2}\right)-\beta_{M}^{\pi N}\left(Q^{2}\right)=\frac{\left(\beta_{M}-\beta_{M}^{\pi N}\right)_{Q^{2}=0}}{\left(1+Q^{2} / \Lambda_{\beta}^{2}\right)^{2}}
$$

The mass scale $\Lambda_{\beta}$ is a free parameter related to the diamagnetism distribution inside the nucleon. It can be extracted from a fit to the VCS data at different $Q^{2}$ values. - Though unsubtracted dispersion relation is valid for the amplitude $F_{2}$ related to the polarizability $P^{(01,01) 0}$ or $\alpha_{E}\left(Q^{2}\right)$, it is particularly relevant to wonder about the quality of the saturation of the subtracted dispersion integrals by $\pi N$ intermediate states only. For this purpose
Table 4. The structure functions determined in the MAMI experiment using the four methods and compared to model predictions at $Q^{2}=0.33 \mathrm{GeV}^{2}$ and $\epsilon=0.62$. The errors are statistical only, except for the first method where two systematic errors are indicated in brackets. The prediction for DR model is given for 2 values of $\Lambda_{\alpha}$ and $\Lambda_{\beta}$ close to the values determined experimentally.

\begin{tabular}{|c|c|c|}
\hline $\begin{array}{c}Q^{2}=0.33 \mathrm{GeV}^{2} \\
\epsilon=0.62\end{array}$ & $\begin{array}{c}\mathbf{P}_{\mathbf{L L}}-\mathbf{P}_{\mathbf{T T}} / \varepsilon \\
\left(\mathrm{GeV}^{-2}\right)\end{array}$ & $\begin{array}{c}\mathbf{P}_{\mathbf{L T}} \\
\left(\mathrm{GeV}^{-2}\right)\end{array}$ \\
\hline $\begin{array}{c}\text { method 1 }[18] \\
\left(\chi^{2}=1.4\right)\end{array}$ & $\begin{array}{c}( \pm .7 \pm 2.2 \\
( \pm 4.3 \pm 0.6)\end{array}$ & $\begin{array}{c}-5.0 \pm .8 \\
( \pm 1.4 \pm 1.1)\end{array}$ \\
\hline $\begin{array}{c}\text { method 2 } \\
\left(\chi^{2}=1.3\right)\end{array}$ & $23.7 \pm 8.1$ & $-7.8 \pm 3.0$ \\
\hline $\begin{array}{c}\text { method 3 } \\
\left(\chi^{2}=1.7\right)\end{array}$ & $33.6 \pm 11.7$ & $-6.5 \pm 4.2$ \\
\hline method 4 & $23.2 \pm 3.0$ & $-3.2 \pm 2.0$ \\
$\left(\chi^{2}=1.5\right)$ & $\left(\Lambda_{\alpha}=1.6 \pm 0.2\right)$ & $\left(\Lambda_{\beta}=0.5 \pm 0.2\right)$ \\
\hline HBChPT $[13]$ & 26.3 & -5.7 \\
\hline LSM [12] & 10.9 & 0.2 \\
\hline ELM [11] & 5.9 & -1.9 \\
\hline NRQCM [9] & 11.0 & -3.5 \\
\hline NRQCM [10] & 14.7 & -4.5 \\
\hline DR [15] & 22.0 & -5.5 \\
& $\left(\Lambda_{\alpha}=1.4 \mathrm{GeV}\right)$ & $\left(\Lambda_{\beta}=0.5 \mathrm{GeV}\right)$ \\
\hline
\end{tabular}

a dipole parametrization has also been proposed:

$$
\alpha_{E}\left(Q^{2}\right)-\alpha_{E}^{\pi N}\left(Q^{2}\right)=\frac{\left(\alpha_{E}-\alpha_{E}^{\pi N}\right)_{Q^{2}=0}}{\left(1+Q^{2} / \Lambda_{\alpha}^{2}\right)^{2}}
$$

The mass scale $\Lambda_{\alpha}$ is the second free parameter of the DR formalism which can be extracted from a fit to the VCS data at different $Q^{2}$ values.

The evolution with the real photon momentum $\mathrm{q}^{\prime}$ of the MAMI VCS data obtained at $Q^{2}=0.33 \mathrm{GeV}^{2}$ is relatively sensitive to the choice of the free parameter values: $\Lambda_{\alpha}=1.6 \pm 0.2 \mathrm{GeV}$ and $\Lambda_{\beta}=0.5 \pm 0.2 \mathrm{GeV}$. The corresponding evolution with the real photon momentum $\mathrm{q}^{\prime}$ is presented by the dotted line in fig 7 . This prediction is rather close to the evolution of method 3 given by the interference between the complete $\mathrm{BH}+\mathrm{Born}$ amplitude and the truncated Non-Born amplitude, except for $\theta$ close to $0^{\circ}$. This kinematical point is only sensitive to $\mathbf{P}_{\mathbf{L T}}$ and this indicates different results for this observable in the framework of these 2 methods.

Results: Table 4 presents the two structure functions in the framework of the four methods and compares them to theoretical predictions presented in the introduction: the heavy-baryon chiral perturbation theory calculation (HBChPT) [13], the linear sigma model (LSM) [12], the effective Lagrangian model (ELM) [11], two non-relativistic constituent quark models (NRQCM) $[9,10]$ and the dispersive relation approach (DR) [15]. The three errors for the first method are, respectively, statistical error on the data, systematic error on photon angular distributions, and systematic error on the normalization. For the other methods only statistical errors are reported. The prediction for the DR model is given for 2 values of $\Lambda_{\alpha}$ and $\Lambda_{\beta}$ close to the values determined experimentally. 

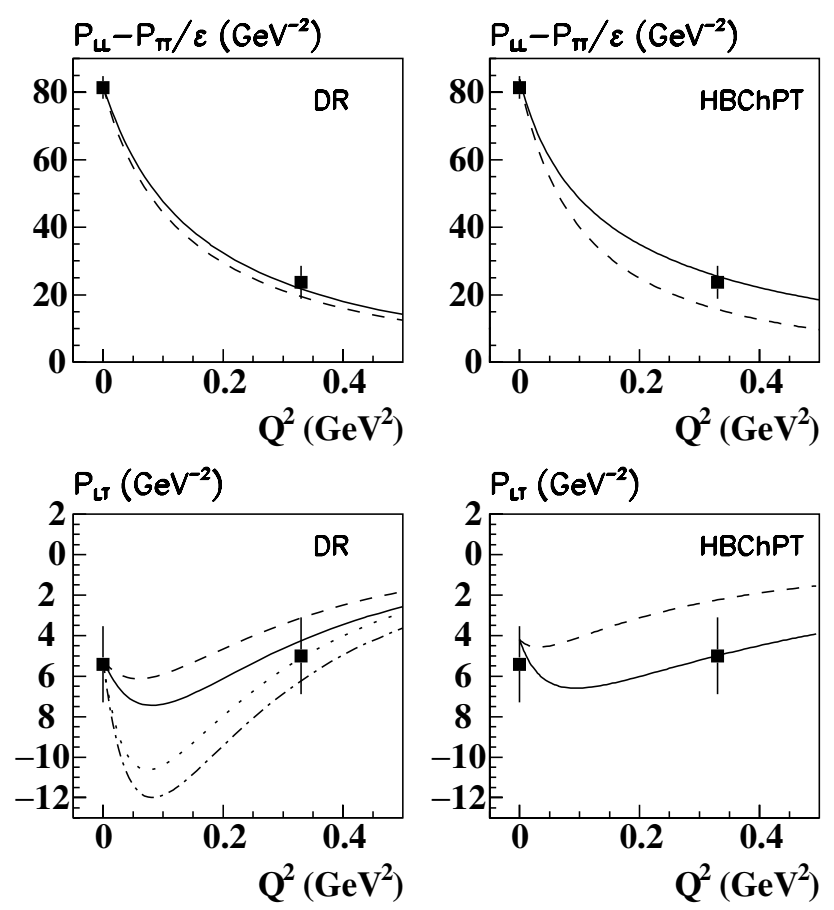

Fig. 9. Comparison of the unpolarized structure functions determined in the VCS MAMI experiment at $Q^{2}=0.33 \mathrm{GeV}^{2}$ and in the RCS results [1] with the predictions of the DR formalism [15] (left panel) and of the $\mathcal{O}\left(p^{3}\right)$ HBChPT [13] (right panel). The upper panels give the result for $\mathbf{P}_{\mathbf{L L}}-\mathbf{P}_{\mathbf{T T}} / \epsilon$ and the lower panel for $\mathbf{P}_{\mathbf{L T}}$. The contributions of the scalar GPs are indicated by the dashed (or dotted) lines and the total contributions of the scalar and spin-dependent GPs are indicated by the solid (or dashed-dotted) lines. The DR prediction for the scalar GP $\alpha_{E}\left(Q^{2}\right)$ is calculated for $\Lambda_{\alpha}=1.4 \mathrm{GeV}$ (upper left panel). Two values of $\Lambda_{\beta}$ are used to calculated the scalar GP $\beta_{M}\left(Q^{2}\right)$ contribution (lower left panel) in order to show the sensitivity. The contributions for $\Lambda_{\beta}=0.4$ and $0.6 \mathrm{GeV}$ are used in the dotted and dashed lines, respectively. Figure extracted from [15].

The first experimental method for which the systematic errors have been carefully studied, gives the two structure functions $\mathbf{P}_{\mathbf{L L}}-\mathbf{P}_{\mathbf{T T}} / \epsilon$ and $\mathbf{P}_{\mathbf{L T}}$ presented in ref. [18]. The three other methods confirm the large values of the two structure functions. The 4 th method in the DR approach is particularly in good agreement with the 1st method for the value of $\mathbf{P}_{\mathbf{L L}}-\mathbf{P}_{\mathbf{T T}} / \epsilon$ and gives a slightly smaller value for $\mathbf{P}_{\mathbf{L T}}$. Only the heavy-baryon chiral perturbation theory calculation (HBChPT) [13] and the dispersive relation approach (DR) [15] predict large values for these two structure functions and seem relevant for the description of the MAMI VCS experiment (see fig. 9).

The structure functions measured in an unpolarized VCS experiment are the sum of two contributions (cf. eq. (7)) : one scalar related to the electric $\alpha_{E}$ and magnetic $\beta_{M}$ polarizabilities measured in RCS and one spindependent. The last contribution vanishes at $Q^{2}=0$. Figure 9 indicates that the effect of the spin GPs is much smaller in the DR calculation [15] than in the $\mathcal{O}\left(p^{3}\right)$ HBChPT [13]. To go beyond, that is to measure indepen- dently all the scalar and spin-dependent polarizabilities, it is necessary to perform a double polarization experiment.

\subsection{The two other unpolarized experiments at JLab and MIT-Bates}

The JLab experiment E93-050 [19] has been performed in the Hall A of the Thomas Jefferson National Accelerator Facility at $Q^{2}=0.9$ and $1.8 \mathrm{GeV}^{2}$. The values of $\epsilon$ are 0.95 and 0.88 , respectively. Data cover the region below the pion threshold and the resonance region up to $\sqrt{s}=2 \mathrm{GeV}$. The experimental analysis of the complete experiment is presented in ref. [19].

The Bates experiment 97-03 [20] has been performed at $Q^{2}=0.05 \mathrm{GeV}^{2}$ and $\epsilon=0.90$. Measurements have been done in-plane and at $90^{\circ}$ out-of-plane, using the OOPS spectrometers. The experiment covers a limited range in polar angle $\theta$ around $90^{\circ}$, so the structure functions are determined from the $\varphi$-dependence of the cross section. Data analysis is still in progress and only preliminary results [20] can be presented. This experiment represents a laboratory achievement, having made the first use of the high duty factor beam in the South Hall Ring and of the full OOPS system.
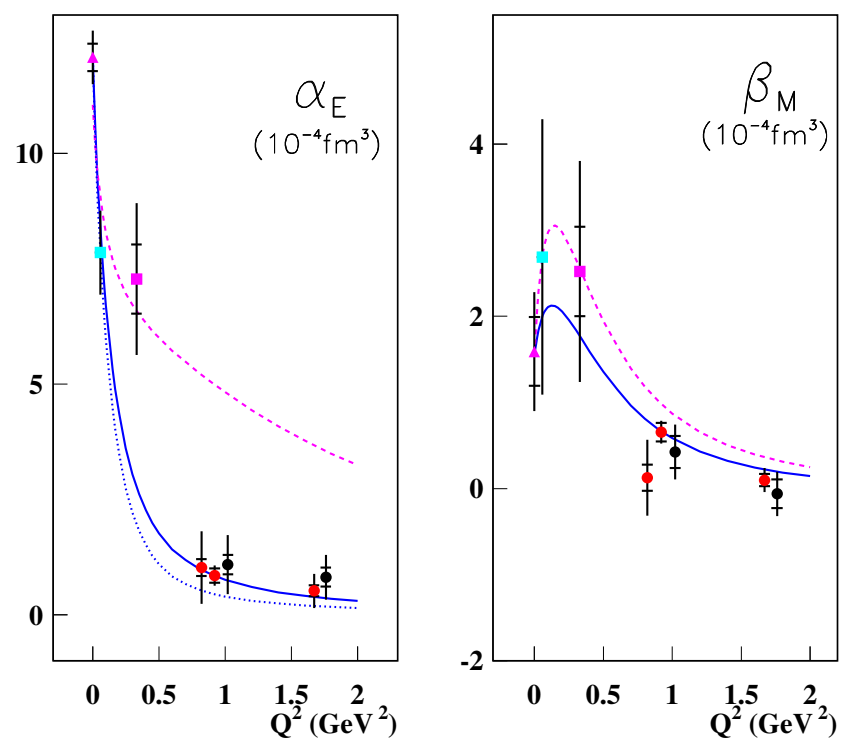

Fig. 10. Compilation of the data on electric $\alpha_{E}\left(Q^{2}\right)$ (left) and magnetic $\beta_{M}\left(Q^{2}\right)$ (right) GPs of the proton. Data points at $Q^{2}=0$ are from ref. [1]. The other points are the analyses of MIT-Bates [20] (still preliminary), of MAMI [18] and different analyses of JLab [19]. JLab points are slightly shifted in abscissa for better visibility. The inner error bars are statistical; the outer ones are the total error. The curves show calculations in the DR model with the values of $\Lambda_{\alpha}$ and $\Lambda_{\beta}$ obtained in each experiment $\left(\Lambda_{\alpha}, \Lambda_{\beta}\right)=(0.70,0.63) \mathrm{GeV}$ in the JLab experiment (solid curve), $(1.60,0.50) \mathrm{GeV}$ in the MAMI experiment (dashed curve) and $(0.60,0.51)$ in the MIT-Bates experiment (dotted curve). Note that the DR predictions for $\beta_{M}\left(Q^{2}\right)$ for the MIT-Bates and MAMI experiments are on the same dotted curve. Figure done thanks to Hélène Fonvieille [26]. 


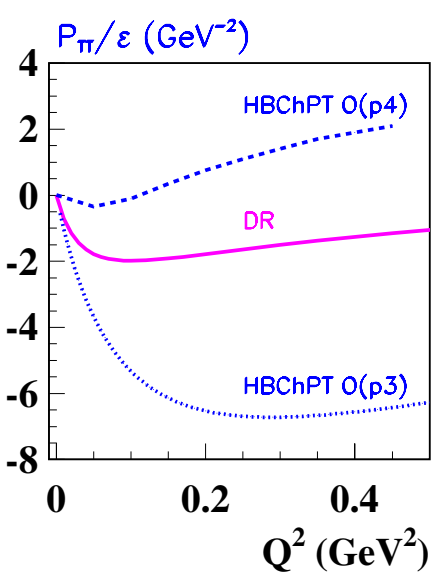

Fig. 11. $\mathbf{P}_{\mathbf{T T}}$ evaluated using the DR model [15] (solid line), the $\mathcal{O}\left(p^{3}\right)$ HBChPT [13] (dotted line) and the $\mathcal{O}\left(p^{4}\right)$ HBChPT [16] (dashed line).

We present in fig. 10 the world results for the electric $\alpha_{E}\left(Q^{2}\right)$ and magnetic $\beta_{M}\left(Q^{2}\right)$ GPs deduced from the MAMI, JLab and MIT-Bates experiments. The value of the electric $\alpha_{E}\left(Q^{2}\right)$ and magnetic $\beta_{M}\left(Q^{2}\right)$ GPs can determined directly by the coefficients $\Lambda_{\alpha}$ and $\Lambda_{\beta}$ obtained in the DR analysis and using eq. (9) and eq. (8) or indirectly by the structure functions $\mathbf{P}_{\mathbf{L L}}-\mathbf{P}_{\mathbf{T T}} / \epsilon$ and $\mathbf{P}_{\mathbf{L T}}$ determined in the LET analysis in which the spin GPs contributions are evaluated in the DR model and subtracted using eq. (7). The agreement between these 2 methods was reasonably controlled in the JLab experiment (see the different results at $Q^{2}=0.9$ and $1.8 \mathrm{GeV}$ ). The direct determination allows us to use also the VCS data in the resonance region (see in fig. 10 the result at $Q^{2}=0.9 \mathrm{GeV}^{2}$ with the smallest statistical error.)

The curves in fig. 10 are calculated using the DR model and the different values of $\Lambda_{\alpha}$ and $\Lambda_{\beta}$ obtained in each experiment. By definition all the DR predictions (see eqs. (9) and (8)) are constrained to go through the experiment RCS point at $Q^{2}=0$. The fact that there is no unique DR curve going through all the data points, especially for the electric polarizability, does not invalidate the model. It simply means that the dipole parametrization of eqs. (9) and (8) does not hold over the entire $Q^{2}$ range. Another fact to be aware of is the model-dependency introduced in this figure by transforming the structure functions into GPs. The spin-dependent GPs are evaluated using the DR model, and as it has been pointed in fig. 9, this evaluation is quite smaller than in the $\mathcal{O}\left(p^{3}\right) \mathrm{HBChPT}[13]$.

It is clear that measurements of individual scalar and spin-dependent GPs are necessary to go further. We can note in fig. 11 the very different predictions for $\mathbf{P}_{\mathbf{T T}}$ using the DR model [15] or the $\mathcal{O}\left(p^{3}\right)$ HBChPT [13] or else the $\mathcal{O}\left(p^{4}\right)$ HBChPT [16]. An extraction of $\mathbf{P}_{\mathbf{T T}}$ can be achieved in a further experiment at MAMI at the same $Q^{2}=0.33 \mathrm{GeV}^{2}$, but with an other value of $\epsilon$. We can take the benefit of the next $1.5 \mathrm{GeV}$ energy of the beam to access a new value of $\epsilon$ to have a comfortable lever arm for a longitudinal-transverse separation.

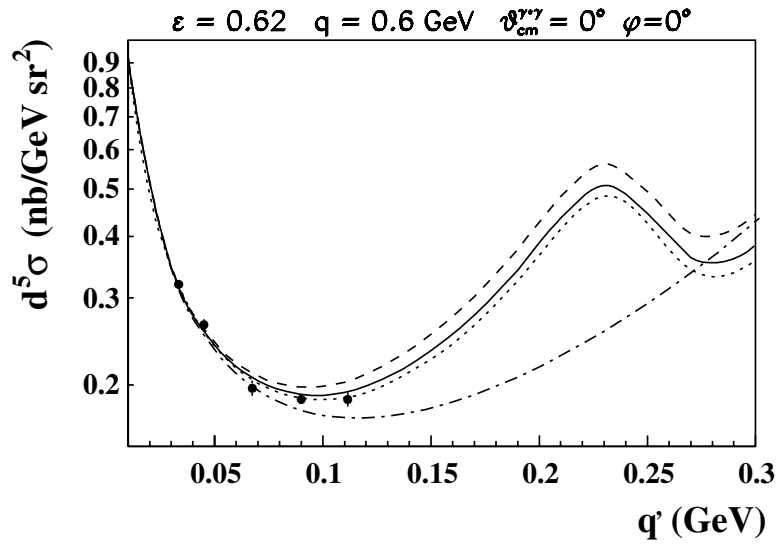

Fig. 12. VCS differential cross section as a function of the real photon energy $q^{\prime}$ in the MAMI kinematics below and above the pion threshold. The VCS MAMI data are reported. The $\mathrm{BH}+$ Born contribution is given by the dash-dotted line. Predictions for the total cross section are given in the DR approach [15] using a fixed value of $\Lambda_{\alpha}=1 \mathrm{GeV}$ and for three values of $\Lambda_{\beta}: 0.6 \mathrm{GeV}$ (solid line); $0.7 \mathrm{GeV}$ (dotted line) and $0.4 \mathrm{GeV}$ (dashed line). Fig extracted from ref. [15].

\section{Single polarized experiments above pion threshold}

Figure 12 shows the DR predictions for photon energies ranging from threshold to the $\Delta$ (1232)-resonance region. The deviation from the $\mathrm{BH}+\mathrm{Born}$ prediction rises strongly after pion threshold. When crossing the pion threshold, the VCS amplitude acquires an imaginary part due to the coupling to the $\pi N$ channel. Therefore single polarization observables become non-zero above pion threshold. A particularly relevant observable is the electron single spin asymmetry (SSA) which is obtained by flipping the electron beam helicity. For VCS this observable is mainly due to the interference of the real $\mathrm{BH}+\mathrm{VCS}$ amplitude with the imaginary part of the VCS amplitude. As the SSA vanishes in-plane, its measurement requires an out-of-plane experiment. Such an experiment has been proposed at MIT-Bates [27] and is being realized at MAMI [28] thanks to one of the spectrometers of the A1 collaboration moving out-of-plane. In fig. 13, the SSA is presented for a kinematics in the $\Delta(1232)$ region, corresponding to $\sqrt{s}=1.2 \mathrm{GeV}$. The DR calculation shows that the SSA is quite sizeable, and it is mainly sensitive to the imaginary part of the VCS amplitude, displaying only a rather weak dependence on the GPs (obtained for the different values of $\Lambda_{\alpha}$ and $\Lambda_{\beta}$ ). Therefore it provides an excellent cross-check of the dispersive input (MAID 2000) in the DR formalism for VCS, in particular by comparing at the same time the pion and photon electroproduction channels through the $\Delta$ excitation. The MAMI analysis is still in progress.

\section{Double polarized experiments at threshold}

A double-polarization VCS experiment is also presently being realized at MAMI. The theoretical framework of 


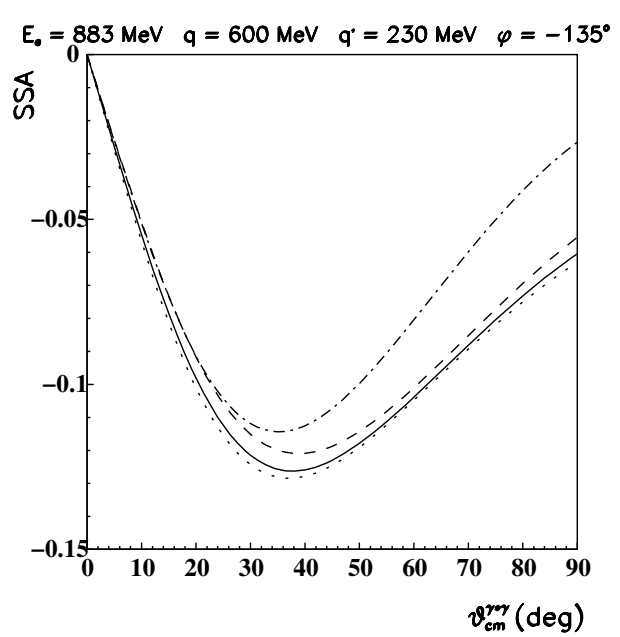

Fig. 13. Electron single-spin asymmetry (SSA) for the VCS MAMI kinematics as a function of the photon scattering angle. The full dispersion results are shown for the values of $\Lambda_{\alpha}=1 \mathrm{GeV}$ and $\Lambda_{\beta}=0.6 \mathrm{GeV}$ (solid curve); $\Lambda_{\alpha}=1 \mathrm{GeV}$ and $\Lambda_{\beta}=0.4 \mathrm{GeV}$ (dashed curve); $\Lambda_{\alpha}=1 \mathrm{GeV}$ and $\Lambda_{\beta}=0.7 \mathrm{GeV}$ (dotted curve) and $\Lambda_{\alpha}=1.4 \mathrm{GeV}$ and $\Lambda_{\beta}=0.4 \mathrm{GeV}$ (dashdotted curve). Figure extracted from ref. [15].

such experiment has been developed by Vanderhaeghen and Guichon $[5,29]$. Below pion production threshold, the VCS amplitude is purely real, and all single-polarization observables are zero. So only double polarization experiment observables can disentangle the GPs. For a polarized electron of pure helicity state $h= \pm 1 / 2$ (longitudinal polarization $\left.\xi_{e}=2 h k / m_{e}\right)$, we can measure the average polarization $\mathcal{P}$ of the recoil proton along the 3 vectors $\left(\boldsymbol{e}_{x_{c m}}, \boldsymbol{e}_{y_{c m}}, \boldsymbol{e}_{z_{c m}}\right)$ associated to the virtual photon direction in the photon-proton CM as defined in fig 14 .

We can define for the 3 axes:

$$
\begin{aligned}
& \mathcal{P} \cdot \boldsymbol{e}(i)= \\
& \frac{\mathrm{d}^{5} \sigma\left[\xi_{e}=\frac{2 h k}{m_{e}}, \boldsymbol{\xi}_{\boldsymbol{p}}=\boldsymbol{e}(i)\right]-\mathrm{d}^{5} \sigma\left[\xi_{e}=\frac{2 h k}{m_{e}}, \boldsymbol{\xi}_{\boldsymbol{p}}=-\boldsymbol{e}(i)\right]}{\mathrm{d}^{5} \sigma\left[\xi_{e}=\frac{2 h k}{m_{e}}, \boldsymbol{\xi}_{\boldsymbol{p}}=\boldsymbol{e}(i)\right]+\mathrm{d}^{5} \sigma\left[\xi_{e}=\frac{2 h k}{m_{e}}, \boldsymbol{\xi}_{\boldsymbol{p}}=-\boldsymbol{e}(i)\right]} \\
& =\frac{\Delta \mathrm{d}^{5} \sigma(h, i)}{2 \cdot \mathrm{d}^{5} \sigma}
\end{aligned}
$$

We obtain a similar low energy prediction as in eq. (5):

$$
\begin{aligned}
\Delta \mathrm{d}^{5} \sigma(h, i) & =\Delta \mathrm{d}^{5} \sigma^{B H+B o r n}(h, i) \\
& +\phi \mathrm{q}^{\prime} \Delta \Psi_{0}(h, i)+\phi \mathcal{O}\left(\mathrm{q}^{\prime 2}\right)
\end{aligned}
$$

So in such a complete experiment we can access 4 observables:

$$
\begin{aligned}
\Psi_{0} & =v_{1}\left(\mathbf{P}_{\mathbf{L L}}-\mathbf{P}_{\mathbf{T T}} / \epsilon\right)+v_{2} \mathbf{P}_{\mathbf{L T}}, \\
\Delta \Psi_{0}(h, z) & =(4 h)\left[v_{1}^{z} \mathbf{P}_{\mathbf{T T}}+v_{2}^{z} \mathbf{P}_{\mathbf{L T}}^{\mathbf{z}}+v_{3}^{z} \mathbf{P}_{\mathbf{L T}}^{\prime \mathbf{z}}\right], \\
\Delta \Psi_{0}(h, x) & =(4 h)\left[v_{1}^{x} \mathbf{P}_{\mathbf{L T}}^{\perp}+v_{2}^{x} \mathbf{P}_{\mathbf{T} \mathbf{T}}^{\perp}+v_{3}^{x} \mathbf{P}_{\mathbf{T T}}^{\prime \perp}+v_{4}^{x} \mathbf{P}_{\mathbf{L T}}^{\prime \perp}\right] \\
\Delta \Psi_{0}(h, y) & =(4 h)\left[v_{1}^{y} \mathbf{P}_{\mathbf{L T}}^{\perp}+v_{2}^{y} \mathbf{P}_{\mathbf{T} \mathbf{T}}^{\perp}+v_{3}^{y} \mathbf{P}_{\mathbf{T T}}^{\prime \perp}+v_{4}^{y} \mathbf{P}_{\mathbf{L T}}^{\perp \perp}\right]
\end{aligned}
$$

Only 6 structure functions are independent: $\mathbf{P}_{\mathbf{L L}}, \mathbf{P}_{\mathbf{T T}}$, $\mathbf{P}_{\mathbf{L T}}, \mathbf{P}_{\mathbf{L T}}^{\mathbf{z}}, \mathbf{P}_{\mathbf{L T}}^{\prime \mathbf{z}}$ and $\mathbf{P}_{\mathbf{L T}}^{\prime \perp} . \mathbf{P}_{\mathbf{L T}}^{\perp}$ is a combination of $\mathbf{P}_{\mathbf{T T}}$

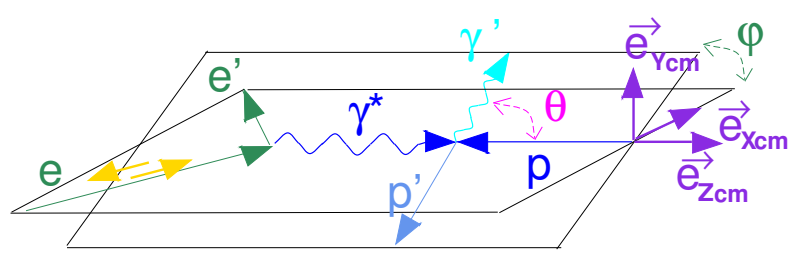

Fig. 14. Kinematics for the $p\left(\boldsymbol{e}, e^{\prime} \boldsymbol{p}^{\prime}\right) \gamma$ reaction.
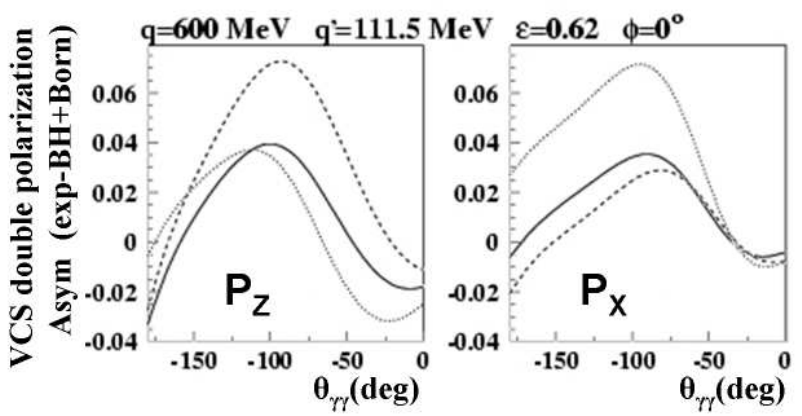

Fig. 15. Predictions for the deviation of the double polarization asymmetry from the $\mathrm{BH}+$ Born contribution using the DR model [15] (solid line), the $\mathcal{O}\left(p^{3}\right)$ HBChPT [13] (dotted line) and the $\mathcal{O}\left(p^{4}\right)$ HBChPT [31] (dashed line).

and $\mathbf{P}_{\mathbf{L L}}, \mathbf{P}_{\mathbf{T} \mathbf{T}}^{\perp}$ a combination of $\mathbf{P}_{\mathbf{L T}}^{\mathbf{z}}$ and $\mathbf{P}_{\mathbf{L T}}, \mathbf{P}_{\mathbf{T T}}^{\prime \perp}$ a combination of $\mathbf{P}_{\mathbf{L T}}^{\prime \mathbf{z}}$ and $\mathbf{P}_{\mathbf{L T}}$.

$\mathbf{P}_{\mathbf{L L}}=a P^{(01,01) 0}$,

$\mathbf{P}_{\mathbf{T T}}=\quad c_{1} P^{(11,11) 1}+c_{2} P^{(01,12) 1}$,

$\mathbf{P}_{\mathbf{L T}}=b P^{(11,11) 0}+c_{3}\left[P^{(11,00) 1}+d_{1} P^{(11,02) 1}\right]$,

$\mathbf{P}_{\mathbf{L T}}^{\mathbf{z}}=\quad c_{4} P^{(11,11) 1}+c_{3}\left[P^{(11,00) 1}+d_{1} P^{(11,02) 1}\right]$,

$\mathbf{P}_{\mathbf{L T}}^{\prime \mathbf{z}}=\quad c_{5} P^{(11,11) 1}+c_{6}\left[P^{(11,00) 1}+d_{1} P^{(11,02) 1}\right]$,

$\mathbf{P}_{\mathbf{L T}}^{\prime}=\quad\left[d_{2} P^{(11,00) 1}+d_{3} P^{(11,02) 1}\right]$.

The $v_{i}^{j}$ in eq. (12) are kinematical factors depending on $\theta$ and $\varphi$. If the out-of-plane angle $\varphi$ remains close to $0^{\circ}$ or $180^{\circ}$, we have the following approximations:

$$
\begin{gathered}
v_{1}=v_{1}^{z} \sim v_{1}^{x} \propto \sin \theta ; v_{2} \sim v_{2}^{z} \sim v_{2}^{x} \sim \text { constant } \\
v_{3}^{z} \sim v_{3}^{x} \propto \cos \theta ; v_{4}^{x} \propto \sin \varphi \sim 0 ; \forall i, v_{i}^{y} \propto \sin \varphi \sim 0 .
\end{gathered}
$$

The GPs can be extracted from the linear system above using the angular distributions of $\Psi_{0}$ and the 3 $\Delta \Psi_{0}(h, i)$. Note that $\mathbf{P}_{\mathbf{L T}}^{\prime \perp}$ can only be extracted by an out-of-plane measurement. Theoretical predictions [31] using the DR model, the $\mathcal{O}\left(p^{3}\right)$ HBChPT and the $\mathcal{O}\left(p^{4}\right)$ HBChPT give a few $\%$ deviation of the double polarization asymmetry from the $\mathrm{BH}+$ Born contribution (see fig. 15).

Such a delicate experiment is being realized using the polarized electron beam available at MAMI and the measurement of the recoil polarization of the outgoing proton in a focal plane polarimeter [30], and the detection of the outgoing electron in the high resolution spectrometer 
moving out-of-plane. It is clearly a very challenging experiment, relying on a very delicate expertise of the complete apparatus and requiring high statistics and very reduced systematic errors.

\section{Conclusion}

An ambitious program to reach the generalized polarizabilies of the proton has been undertaken at MAMI over the last ten years. The ultimate Grail is the separation between spin-independent and spin-dependent GPs which seems very promising for the study of the nucleon structure.

All the results reported here are the fruits of the complete A1-VCS collaboration. I would like to acknowledge all the students, Luca Doria, Peter Janssens, Imad Bensafa, Jan Friedrich, Julie Roche, David Lhuillier, Dominique Marchand for which the work was essential to produce reliable results on these very meticulous experiments. I wish to underline the strong support and the synergy given by Hélène Fonvieille, Harald Merkel, Michael Distler, Luc Van Hoorebeke, Gabriel Tamas, Robert Van de Vyver, Jörg Friedrich, Thomas Walcher for this research. I am also very grateful to Dieter Drechsel, Pierre Guichon, Marc Vanderhaeghen, Barbara Pasquini, Stefan Scherer, Thomas Hemmert, Ulf Meissner for their pedagogical lectures and theoretical support in the data interpretation.

It is clear that the success of the VCS MAMI experiments has its origin in the coherent effort between excellent physicists as Karl-Heinz Kaiser, always concerned with the performance and the high quality of the electron facility, Thomas Walcher strongly supporting and managing all the efforts for such a challenging experimental program, Jörg Friedrich, expert of delicate and precise experiments and Dieter Drechsel, stimulating progress in our scientific knowledge.

\section{References}

1. V. Olmos de León et al., Eur. Phys. J. A 10, 207 (2001).

2. Y.S. Tsai, Phys. Rev. 122, 1898 (1961).

3. H. Arenhövel, D. Drechsel, Nucl. Phys. A 233, 153 (1974).

4. P.A.M. Guichon, G.Q. Liu, A.W. Thomas, Nucl. Phys. A 591, 606 (1995).

5. P.A.M. Guichon, M. Vanderhaeghen, Prog. Part. Nucl. Phys. 41, 125 (1998).
6. D. Drechsel, G. Knöchlein, A. Metz, S. Scherer, Phys. Rev. C 55, 424 (1997).

7. D. Drechsel, G. Knöchlein, A. Yu Korchin, A. Metz, S. Scherer, Phys. Rev. C 57, 941 (1998).

8. F.E. Low, Phys. Rev. 110, 974 (1958)

9. G.Q. Liu, A.W. Thomas, P.A.M. Guichon, Austral J. Phys. 49, 905 (1996).

10. B. Pasquini, S. Scherer, D. Drechsel, Phys. Rev. C 63, 025205 (2001).

11. M. Vanderhaeghen, Phys. Lett. B 368, 13 (1996).

12. A. Metz, D. Drechsel, Z. Phys. A 356, 351 (1996); 359, 165 (1997).

13. T.R. Hemmert, B.R. Holstein, G. Knöchlein, S. Scherer, Phys. Rev. D 55, 2630 (1997); Phys. Rev. Lett. 79, 22 (1997); T.R. Hemmert, B.R. Holstein, G. Knöchlein, D. Drechsel, Phys. Rev. D 62, 014013 (2000).

14. B. Pasquini, M. Gorchtein, A. Metz, M. Vanderhaeghen, Eur. Phys. J. A 11, 185 (2001).

15. D. Drechsel, B. Pasquini, M. Vanderhaeghen, Phys. Rep. 378, 99 (2003).

16. C.W. Kao, M. Vanderhaeghen, Phys. Rev. Lett. 89, 272002 (2002).

17. V. Bernard, N. Kaiser, A. Schmidt, U. Meissner, Phys. Lett. B 319, 269 (1993); Z. Phys. A 348, 317 (1994).

18. J. Roche et al., Phys. Rev. Lett. 85, 708 (2000).

19. G. Laveissiere et al., Phys. Rev. Lett. 93, 122001 (2004).

20. J. Shaw, R. Miskimen, MIT-Bates Proposal 97-03, (1997) and P. Bourgeois, PhD Thesis.

21. K.I. Blomqvist et al., Nucl. Instrum. Methods A 403, 263 (1998).

22. G. Höhler, E. Pietarinen, I. Sabba-Stefanescu, F. Borkowski, G.G. Simon, V.H. Walther, R.D. Wendling, Nucl. Phys. B 114, 505 (1976); private communication.

23. M. Vanderhaeghen, J.M. Friedrich, D. Lhuillier, D. Marchand, L. Van Hoorebeke, J. Van de Wiele, Phys. Rev. C 62, 025501 (2000).

24. P. Janssens, L. Van Hoorebeke et al., to be published in Nucl. Instrum. Methods.

25. D. Drechsel, O. Hanstein, S.S. Kamalov, L. Tiator, Nucl. Phys. A 645, 145 (1999).

26. H. Fonvieille, Proceedings of the Erice School of Nuclear Physics, 26th Course, Prog. Part. Nucl. Phys. 55, 198 (2005) and private communication.

27. N.I. Kaloskamis, C.N. Papanicolas, MIT-Bates proposal (1997).

28. N. d'Hose, H. Merkel, MAMI Proposal (2001).

29. M. Vanderhaeghen, Phys. Lett. B 402, 243 (1997).

30. Th. Pospischil et al., Nucl. Instrum. Methods A 483, 726 (2002).

31. C.W. Kao, B. Pasquini, M. Vanderhaeghen, Phys. Rev. D 70, 114004 (2004). 